\title{
The only way for minority cultural survival
}

\author{
Asymmetric social boundaries allow a minority culture to reap the benefits of outside interaction while maintaining \\ its distinctiveness. This opens questions about the nature of intergroup interactions and whether such boundaries \\ are the only way to preserve valued cultural norms.
}

\section{Adrian Viliami Bell}

W e live in the Age of Interaction ${ }^{1}$. Human groups' experience with each other has created the tangled cultural repertoire that we see increasing in frequency today. Our diverse languages, foods, music, dress and life events reflect the human species' unique ability to develop, give and take. These domains of human life are also the battlegrounds by which we witness the immense cultural losses of recent. For minority communities, language extinction is a heavy concern, and dance and food are protected as a group's public expression of their distinct identity. If the global trends are irreversible, as they appear so, what will prevent or minimize the loss of distinct cultural practices? In other words, how does minority culture survive in this expanded Age of Interaction.

In a provocative theoretical study in Nature Human Behaviour, Bunce and McElreath ${ }^{2}$ detail individual-level mechanisms by which cultural norms in a less-powerful minority group are maintained. Using a mathematical modelling tradition that treats norm evolution as variants of a coordination game $e^{3,4}$, they encourage intergroup interactions by making them profitable. However, one group receives greater absolute benefits from the between-group interaction than the other. This assumption simulates group differences in power - the seashells-for-gold relationships that characterize much of the past industrial, colonial and contemporary global expansion. This power imbalance often means the arrows of cultural loss are disproportionately experienced by the small and impoverished. Thus, the question is not only about the numerical disadvantage of the minority group, but also about a lesspowerful minority group. In this context, is there a mechanism for the minority group to preserve its cultural norms?

For those who wish for both integration and cultural preservation, the results are not especially encouraging. Assortment and group boundaries appear as prerequisites for the minority culture to survive. Specifically, in the case of frequent majority-minority intergroup interactions, there must be a culturally conservative intragroup interaction that favours minority norms. That is, if a minority group member greets someone in their group, it should on-average be with someone with the same cultural norms. This means majority group members do not occupy spaces within the minority group.

This mathematical result may reflect the minority clubs, schools, and other formal and informal organizations that pass down cultural norms in majority-dominated contexts. It embodies the role of ethnic enclaves as forces of cultural conservation. However, assimilation studies in urban environments often show significant spatial mobility of immigrants as they begin to occupy majority spaces over generations ${ }^{5,6}$. This may mean that a hard boundary may not be needed to protect minority norms if the majority norm becomes not too different. Further, the cultural norms of many urban dwellers result from negotiating multi-ethnic group interactions. Hence, the assimilation pressure put on by the majority, if there is one, may be diffused across multiple groups. For Bunce and McElreath's results to generalize across more complex scenarios representing much of the minority experience, a little more modelling is needed.

Despite these musings, Bunce and McElreath's attempt at modelling the survival of minority culture is very welcome. Because they were empirically motivated by a specific ethnographic case, between the Mestizos and Matsigenka of the Amazon, their study is admirably tied to a very relevant question. The boundary requirement to protect valued minority cultural norms appears sound. However, more work is needed to address the larger question concerning many vulnerable groups: Is this the only way?

\section{Adrian Viliami Bell}

Department of Anthropology, University of Utah, Salt Lake City, UT, USA.

e-mail:adrian.bell@anthro.utah.edu

Published online: 26 February 2018

https://doi.org/10.1038/s41562-018-0313-8

\section{References}

1. Castles, S. \& Miller, M. The Age of Migration (Palgrave MacMillan, New York, NY, 2014)

2. Bunce, J. \& McElreath, R. Nat. Hum. Behav. https://doi. org/10.1038/s41562-018-0306-7 (2018).

3. McElreath, R., Boyd, R. \& Richerson, P. J. Curr. Anthropol. 44, 122-129 (2003).

4. Boyd, R. \& Richerson, P. J. Cult. Anthropol 2, 65-79 (1987)

5. South, S. J., Crowder, K. \& Chavez, E. Demography 42 497-521 (2005).

6. Simpson, L. \& Finney, N. Popul. Space Place 15, 37-56 (2009). 